\title{
Adaptation and reorganisation of primary care facility during COVID-19 pandemic: a perspective from primary health care corporation, Qatar
}

\author{
Abdul Bari Shaik*, Noor Fathima Shaik, Shawqiya Salman Al Majid, Sanaulla Sheik
}

Department of Primary Health Care, Primary Health Care Corporation, Doha, Qatar

Received: 30 November 2020

Revised: 09 December 2020

Accepted: 11 December 2020

*Correspondence:

Dr. Abdul Bari Shaik,

E-mail: docshaik@yahoo.com

Copyright: (C) the author(s), publisher and licensee Medip Academy. This is an open-access article distributed under the terms of the Creative Commons Attribution Non-Commercial License, which permits unrestricted non-commercial use, distribution, and reproduction in any medium, provided the original work is properly cited.

\begin{abstract}
The COVID-19 pandemic has brought unprecedented challenges to healthcare systems across the world. It has had a devastating impact on peoples' health, well-being and livelihood, crippling the economy, aviation, travel, hospitality and various other sectors. In order to curb its impact various governments had to take drastic measures to deal with this crisis. In Qatar, the major healthcare providers led by the MOPH responded swiftly by implementing major changes. They developed infrastructure, revisited public health policies, modified their operations and functioning which helped to cope with this evolving public health emergency. This paper reviews how a small health center in Qatar had to adapt its services, infrastructure and pathways to the changing needs and demands in a timely fashion leading to improved patient care and helping to contain the outbreak in both patients and staff alike. It also evaluates how Primary health care corporation at an organization level paved the transition in every aspect to ensure efficient and safe delivery of primary care services to everyone. As the world is preparing to overcome the pandemic, the paper highlights the value of teamwork in responding to the adversity by creating innovative ways of working and how good leadership can have a positive influence on healthcare and society in general.
\end{abstract}

Keywords: COVID-19, Primary health care Corporation, Umm Ghuwailina health centre, Qatar, Ministry of public health

\section{INTRODUCTION}

COVID-19 is a disease that is caused by the novel corona virus, subsequently named as "severe acute respiratory syndrome coronavirus 2 (SARS-CoV-2)" was first reported in Wuhan city, China in December 2019. ${ }^{1}$ The first case outside China was reported in Thailand on 13th January 2020. ${ }^{2}$ At the end of January 2020 The World health organization (WHO) announced it as a public health emergency of international concern. Following the rise in cases across the world the WHO on 11th March 2020 declared COVID-19 as a pandemic. ${ }^{3}$ This led to unprecedented challenges at a global scale to public health, economy, society and all aspects of peoples' lives.
Due to widespread lockdowns, borders closing and quarantine measures to contain the spread of virus, unemployment increased pushing many to poverty as they continued to struggle to access basic services. The pandemic had a devastating impact worldwide with over 58 million reported cases of infections and well over 1.3 million deaths as a direct result of the COVID-19 infections.

The state of Qatar is a small country along the northeastern coast of the Arabian Peninsula. It is one of the richest countries in the world with a diverse population of around 2.8 million. The first case of COVID-19 in Qatar was confirmed on 29th February 2020. At the onset of the 
pandemic, The Ministry of public health in Qatar (MOPH) responded swiftly by developing a COVID-19 Qatar National response action plan in order to ensure that the health, well-being and prosperity of everyone in the country is protected. It took commendable measures to prepare, monitor, respond and recover from the COVID-19 pandemic. $^{3}$ The MOPH in collaboration with other government-funded major stakeholders like Hamad medical corporation and primary health care corporation (PHCC) were tasked to manage the rapidly escalating crisis, prevent the spread of infection, mitigate risks, control and contain the disease while continuing to provide the highest possible care for the people of Qatar.

As per the current MOPH data the Government Health Sector has performed over 1.1 million COVID-19 RT PCR swabs, of them 138,648 were positives and 1,35,862 recovered as of 30th November 2020. Figure 1 compares the number of cases in Qatar with other countries. Despite the rising number of COVID-19 cases in the country the vast majority of them were asymptomatic who recovered completely. This has been ascribed to the younger age group (20-49 years) of those affected, as majority of expatriate craftsmen and manual workers account for about $60 \%$ of Qatar's total population. ${ }^{4}$ Till date 237 people have lost their lives due to COVID-19 infection which is among the lowest mortality rates in the world. Figure 2 shows the mortality rates due to COVID-19 in Qatar in comparison with other countries. This is attributed largely to the timely coordinated government response at a national level, high standards of healthcare services accessible to all residents without charge, optimal critical care infrastructure, widespread testing, national policies such as closure of schools and certain workplaces, travel restrictions, social distancing, mandatory wearing of masks in public areas, promoting public awareness and introduction of Ehteraz application linked to COVID-19 testing and contact tracing.

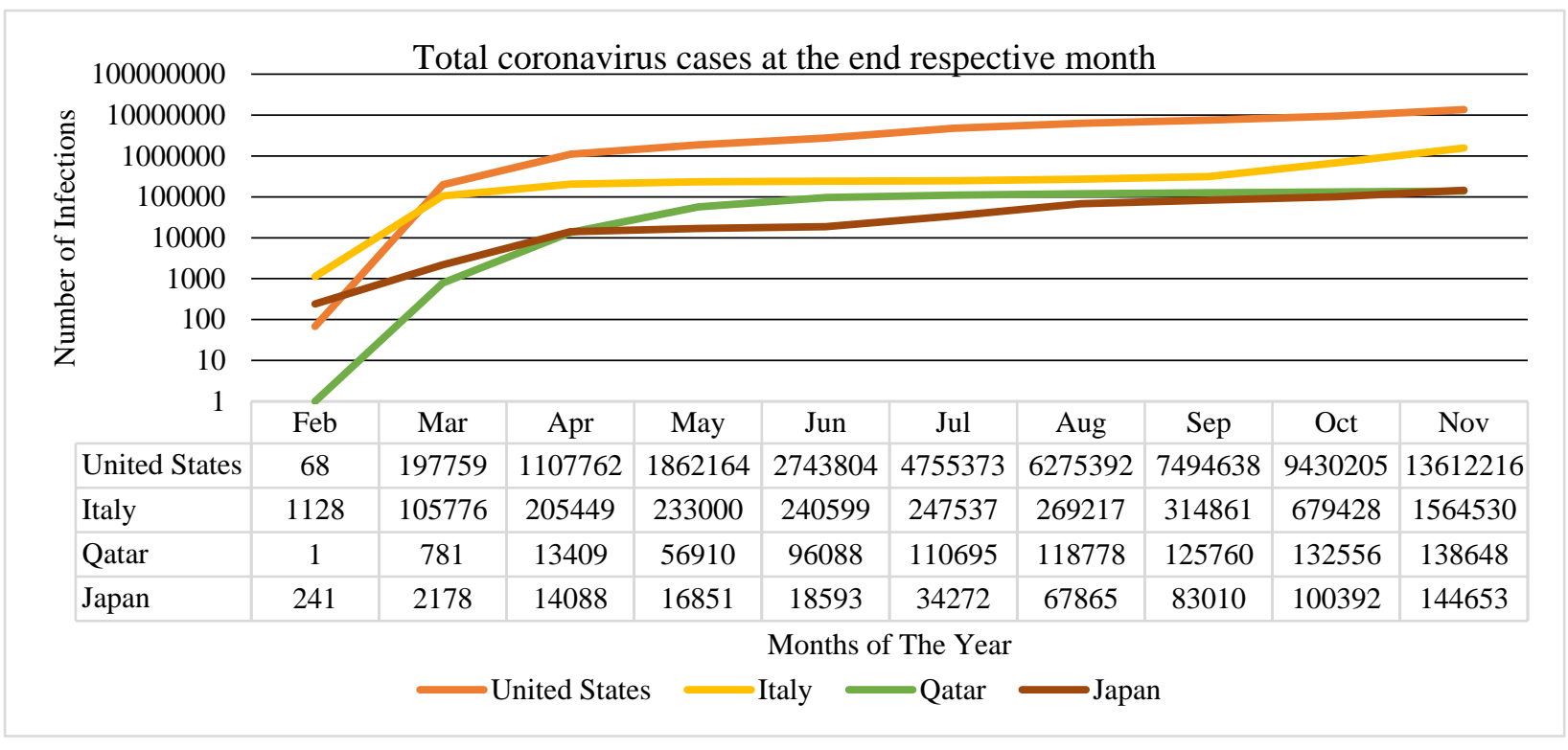

Figure 1: Total COVID 19 cases at the end of each month from February to November 2020 coronavirus update (Live): 63,121,997 Cases and 1,465,919 Deaths from COVID-19 virus pandemic - worldometer (worldometers.info). ${ }^{5}$

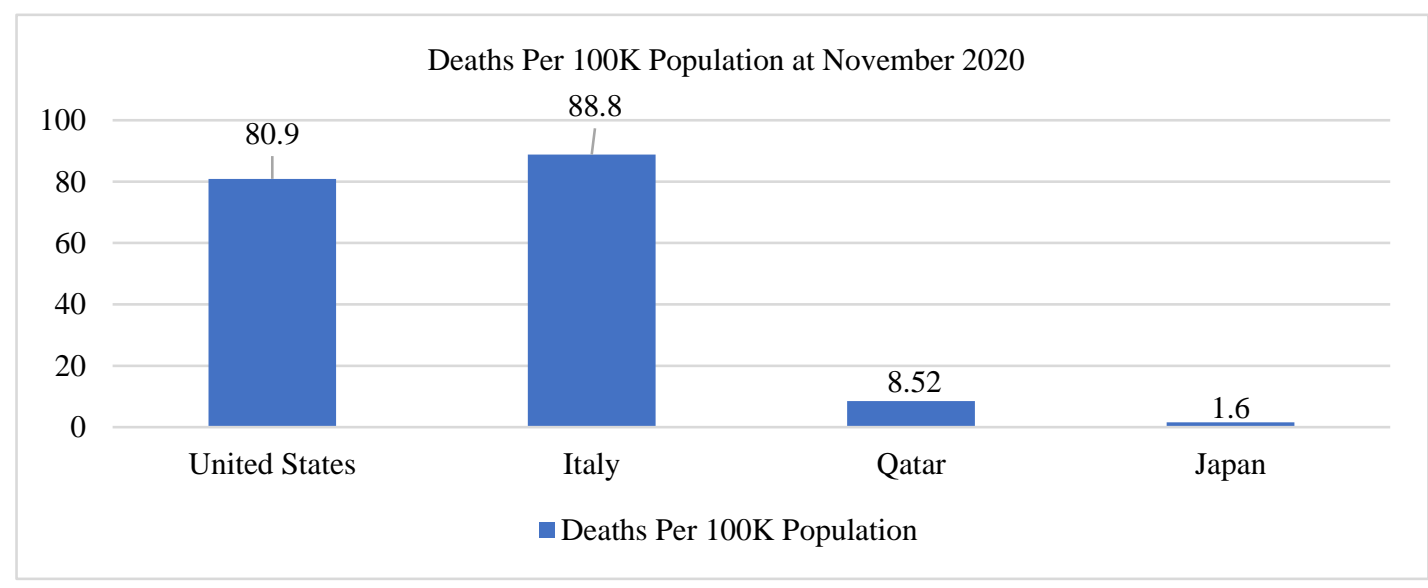

Figure 2: Deaths per 100,000 population due to covid-19 infection countrywise at November 2020 (mortality analyses - Johns Hopkins Coronavirus Resource Center (jhu.edu). ${ }^{6}$ 
PHCC is the leading primary care provider in Qatar with a network of 27 primary health care centres across the country. Our focus in this review is Umm Ghuwailina health centre (UMG) which is one of the PHCC centres and how it responded to the crisis by modifying services and pathways to better manage the challenges and continue to provide primary care services to those in need. UMG is a small but busy health center based in central Doha catering to over 60,000 registered patients. It provides a broad spectrum of services ranging from routine general medical care, family medicine clinics, non-communicable disease clinics (NCD), well woman, vaccination, ante-natal, post-natal clinics, school health reviews, social workers, dental clinics, laboratory service and an on-site pharmacy.

\section{WHY THE CHANGE WAS NEEDED?}

The global rise in number of COVID infections has put health systems under immense pressure to respond to this public health emergency and to minimise its impact. Healthcare services had to adapt and modify their systems and functioning. PHCC did so by redeploying four of its centres as dedicated COVID test and hold centres and channelling their existing patient population to other nearby health centres within catchment in order to continue providing routine services and reduce the burden on already stretched resources. Umm Ghuwailina Health centre was no exception and had to reconsider its protocols and policies to provide primary care services in addition to improvising and accommodating the challenges posed by the pandemic. The limited capacity within the health centre, temporary increase in patient population, lack of restrictions on patient movement in the premises along with a steady increase in the COVID19 cases was impacting the waiting times for patients and inadvertently increasing the risk of acquiring infection for patients and healthcare workers leading to serious staffing concerns. Managing change can be a complex and demanding process, to make it relevant and worthwhile it should be evaluated, planned and implemented well. ${ }^{7}$ The unpredictability associated with this evolving pandemic necessitated an urgent need for constant risk assessment and pertinent changes to improve patient care.

\section{CHANGES IMPLEMENTED AT HEALTH CENTRE}

Prior to COVID-19 pandemic, UMG Health centre did not have zones and patients could move within the health centre without any restrictions. Patients arriving at the health centre would meet greeter nurse who assessed their reason for attendance and guided them to the required services. The receptionist would register the patient and direct them to the triage nursing station where they would be assessed for their clinical needs. As per the triage policy the patients would then be categorized as routine, priority or urgent cases and are dealt with accordingly by the treating physician. The pivotal changes brought about post COVID was the creation of red and green zones in the health centre and a "Swabbing window" with an intention of segregating high-risk patients and optimizing the use of health care resources. The rationale behind Red and Green zones was to prevent outbreaks by limiting person-to-person transmission and promptly managing the suspected cases.

The "green zone" dealt with all non-COVID cases and routine patients while the "red zone" was for those patients who met the case definition criteria of COVID19 infection and asymptomatic patients having direct contact with positive cases. The routine patients in the green zone would be seen by the fully PPE donned Greeter nurse for temperature check, quick review and directed to the appropriate area based on presentation. Patients with trivial queries and concerns were advised to either ring 107 phone line to book a telephone consultation with the physician or ring 16000 call centre for a phone or video consultation with a nurse and physician. The triaging nurse then prioritizes the visit based on the need for appropriate management.

Patients in the red zone would be attended by a 'greeter nurse' who is donned in full PPE including N95 mask. Following a quick initial assessment based on 'visual triage questionnaire' relevant to covid-19 if the patient appears unwell, they would be taken to the 'isolation room' for urgent assessment by the physician. All other patients would be directed to the triage room for vital signs check and if found to have abnormal vital parameters would be directed to isolation room for further assessment. Those who are clinically stable, with mild symptoms or asymptomatic would be given a choice of waiting in their own vehicle in health centre parking or appropriate reception waiting area (one for those with symptoms and another for asymptomatic patients). It was noted that most patients preferred to wait in their vehicle whilst awaiting further assessment or swab testing which helped to reduce overcrowding.

The other significant change the health centre made was the creation of a 'swabbing window' for COVID-19 testing and screening. This was done by structurally altering the window within a consultation room facing the car park to allow swabbing without the need for patients to enter the main building or isolation room. A physical barrier kept outside the window allowed for privacy of those being swabbed. This change was needed for a couple of reasons: Firstly, at the onset of pandemic, many staff in the health centre were tested positive for covid-19 leading to staff shortage with a potential to disrupt normal functioning of the centre and compromising patient care if unchecked. Secondly, the 'swabbing window' dramatically decreased the patient waiting times by reducing the need for disinfection procedures of isolation room. To address these concerns the health centre management came up with this innovative idea to tackle the problem without compromising patient care. The 'swabbing window' was unique to UMG among the 27 PHCC health centres and this was appreciated by the 
managing director and the executive team during their leadership walkaround. All these measures resulted in better management of patient flow and minimized the risk of cross-infection leading to improved patient access, patient care and overall patient satisfaction.

At an organisation level the PHCC has responded rapidly to the pandemic by making significant changes to its normal functioning by transforming 4 large health centres completely into COVID testing and holding centres, reducing some of the non-urgent appointments but maintaining urgent and walk-in-clinic, laboratory, pharmacy and diagnostic services. It has also set up a call centre with dedicated nurses and doctors for daily telephone and video consultations. ${ }^{8}$

Implemented home delivery of chronic medications to avoid the need to visit the health centre. Initiated telephone consultation services in all health centres, as it became primary mode of communication for all routine (non-urgent) patients who walked into HC, pre-booked family medicine model (FMM) clinic appointments, virtual diabetes clinic (VDC), non-communicable disease (NCD), ante-natal clinics till 24 weeks gestation, dietitian and health educator clinics. Nurses called patients on a daily basis to communicate COVID-19 swab results and to review of home-quarantine patients daily.

\section{DISCUSSION}

COVID-19 pandemic is the worst international public health emergency of this century affecting not just health but social, economic and every dimension of human life across the world.9 Qatar was no different, but despite high number of infections it was ranked among the lowest case fatality rates in the world. This was possible due to its preplanning and swift response at the onset of pandemic. The Government rapidly expanded hospital capacity by creating new facilities for 37,000 isolation beds and 12,500 quarantine beds and invested heavily in infrastructure, economy and adopting significant public health policy changes to support its people and manage evolving crisis.10 This reaffirms the fact that good governance is the key to improve performance in the heath sector. ${ }^{11}$ The Ministry of Public Health along with the primary health care corporation and other major providers worked together at various levels in coordinating and managing the services. Organisations are known to contribute to quality improvement through participation in major system changes, integrating health and social care systems, engaging and communicating with all stakeholders, building culture of trust, supporting innovation and problem solving, this was noted here within major health service providers. ${ }^{12}$

Following the changes to the patient pathway in Umm Ghuwailina Health Centre with creation of zones and 'swabbing window' there was a huge reduction in COVID-19 positive staff members, and the waiting times for those patients awaiting swabbing was cut significantly leading to much smoother and efficient patient flow within the health centre. Though simple changes may not always lead to big impact, they can consistently drive performance forward, ease implementation and sustainability in the long run. By taking prompt action and fixing trivial problems, an organisation strives to develop a problem-solving culture which can ultimately lead to large scale solutions. ${ }^{13}$ This was a positive experience appreciated by the staff and patients' which not just helped to ease the whole process but also minimised everyone's risk of exposure. A team will function well if every member communicates effectively to merge their observations, expertise and decisionmaking responsibilities to optimise patient care. ${ }^{14}$ Initially the frontline healthcare staff were apprehensive not knowing how this would impact their already stressful work and how patients would react, but their commitment to give their best for patient care and team spirit made the whole transition process worthwhile.

The health centre management and administrators should be credited for taking proactive steps during such uncertain and testing times, making sure every team member is part of this change with a common goal to improving the service and functioning with the limited resources. Managers at different levels play a key role facilitating, training, coordinating and focussing on fostering a culture of clinical excellence and articulating the organisational culture to their staff. ${ }^{15}$ UMG became a model of change in PHCC with timely interventions and providing strategic leadership from within the health centre. Throughout its journey it was supported by the organisation enabling it to deal with the areas of concern promptly and establishing itself as a safe and effective healthcare facility. This is possible when employees understand the origin and rationale behind the organisational policies and can easily comprehend and comply with them. ${ }^{16}$ As we prepare to turn over a new leaf, the success story of UMG health centre and its workplace modifications during the peak of the pandemic is a true testament for the hard work and dedication of all their workforce which was well led by an efficient management team.

\section{CONCLUSION}

COVID-19 pandemic has continued to have a devastating impact on peoples' life across the world. Healthcare systems have particularly taken the strain and had to adapt quickly in order to contain and manage this international public health emergency. Qatar has been at the forefront of this change and primary health care corporation has stood up to the challenge by being proactive in modifying and reshaping healthcare services as per the demands in this extremely challenging phase and delivered high quality urgent and primary care services working in collaboration with other major Governmental agencies. Umm Ghuwailina health centre, being a small facility has demonstrated courage and commitment by taking prompt measures and proving that 
teamwork and collaboration can overcome any adversity. This pandemic has hit us hard and has triggered a steep learning curve enabling us to unify, broaden our horizons and remain hopeful of a better tomorrow.

Funding: No funding sources

Conflict of interest: None declared

Ethical approval: Not required

\section{REFERENCES}

1. World Health Organization. Infection prevention and control during health care when COVID-19 is suspected: interim guidance, 19 March 2020. World Health Organization; 2020. Available at: https://www.who.int/publications/i/item/10665331495. Accessed on 6 November 2020.

2. World Health Organization. Archived: WHO Timeline -COVID-19. Available at: https://www.who.int/news/item/27-04-2020-whotimeline---COVID-19. Accessed on 7 November 2020.

3. Ministry of Public Health. Coronavirus Disease 2019 (COVID-19) Updates. Qatar, 2020. Available at: https://covid19.moph.gov.qa/EN/Pages/ default.aspx Accessed on 08 November 2020.

4. Jeremijenko A, Chemaitelly $\mathrm{H}$, Ayoub $\mathrm{HH}$, Abdulla MA, Abou-Samra AB, Al Ajmi JA, et al. Evidence for and level of herd immunity against SARS-CoV2 infection: the ten-community study.

5. Total COVID 19 cases at the end of each month from February to November 2020 Coronavirus Update (Live): 63,121,997 Cases and 1,465,919 Deaths from COVID-19 Virus Pandemic Worldometer. Available at: worldometers.info. Accessed on 30 November 2020.

6. Deaths per 100,000 Population due to Covid-19 infection countrywise at November 2020 (Mortality Analyses - Johns Hopkins Coronavirus Resource Center. Available at: jhu.edu. Accessed 29 November 2020.
7. Al-Abri R. Managing change in healthcare. Oman Medic J. 2007;22(3):9.

8. Al Kuwari M, Abdulmalik M, Al Abdulla S, Bakri AH, Gibb J, Kandy M. The COVID-19 Pandemic Impact on Primary Health Care services: An Experience from Qatar. medRxiv. 2020.

9. Revenga A, Galindo J. Responding to global systemic shocks: applying lessons from previous crises to Covid-19. Global agenda. 2020;30:25.

10. Al Khal A, Al-Kaabi S, Checketts RJ. Qatar's response to COVID-19 pandemic. Heart Views. 2020;21(3):129.

11. Fryatt R, Bennett S, Soucat A. Health sector governance: should we be investing more? Bio Med J Glob Heal. 2017;2(2).

12. Fulop NJ, Ramsay AI. How organisations contribute to improving the quality of healthcare. Bio Med J. $2019 ; 365$.

13. The Case for Making Simple Changes [Internet]. Healthleadersmedia.com. 2020. Available at: https://www.healthleadersmedia.com/nursing/casemaking-simple-changes. Accessed on 20 November 2020.

14. World Health Organization. Being an effective team player. World Health Organization (Ed.), Patient safety curriculum guide. Multi-professional edition. 2011:133-51.

15. Saint S, Kowalski CP, Banaszak-Holl J, Forman J, Damschroder L, Krein SL. The importance of leadership in preventing healthcare-associated infection: results of a multisite qualitative study. Infect Cont Hospit Epidemiol. 2010;31(9):901.

16. O'Donnell J, Vogenberg FR. Policies and procedures: enhancing pharmacy practice and limiting risk. Pharm Therapeut. 2012;37(6):341.

Cite this article as: Shaik AB, Shaik NF, Al Majid SS, Sheik S. Adaptation and reorganisation of primary care facility during COVID-19 pandemic: a perspective from primary health care corporation, Qatar. Int J Community Med Public Health 2021;8:434-8. 